\title{
Revisión sobre el dolor neuropático en el síndrome del pie diabético
}

\author{
M. C. MARTÍN MUÑOZ, M. E. ALBARRÁN JUAN', E. M. LUMBRERAS MARÍN² \\ C. S. Calesas. Área 11. ${ }^{~}$ C. S. Presentación Sabio. Área 8. Madrid. ${ }^{2}$ Hospital de Getafe. \\ Madrid
}

\section{RESUMEN}

La diabetes mellitus (DM) es la causa más común de neuropatía en el mundo occidental. Se ha estimado una prevalencia del $28,5 \%$, que varía en función de las medidas utilizadas para definir la neuropatía diabética. Un rápido reconocimiento y una adecuada atención de los factores de riesgo que condicionan el pie diabético pueden prevenir o retardar la aparición de complicaciones.

La generación de dolor neuropático no se debe sólo a causas biológicas, sino que están involucradas respuestas físicas y emocionales. La neuropatía puede conducir a la amputación por varios caminos, los cuales incluyen la pérdida de alguna de las funciones de los nervios periféricos.

El control de la glucemia, de los síntomas, el cuidado de los pies son estándares de referencia e involucran a todo el equipo de salud dedicado a la atención integral de los pacientes con DM. El conocimiento de los mecanismos patogénicos abre una puerta para futuros tratamientos. Probablemente sean éstos más efectivos cuanto más tempranamente se apliquen en el curso de la neuropatía, cuando la afectación nerviosa es reversible todavía.

PALABRAS CLAVE: Diabetes mellitus. Pie diabético. Dolor neuropático. Etiología. Tratamiento.
PAINFUL NEUROPATHY IN THE DIABETIC FOOT SYNDROME: A REVIEW

\section{ABSTRACT}

Diabetes mellitus (DM) is the most common cause of neuropathy in the western world. Recent studies estimate prevalence around $28,5 \%$ but results vary depending on measure used to define diabetic neuropathy. Early detection and carefull attention to risk factors determining diabetic foot may avoid or delay complications.

Pain generation is due to biologic causes as well as physical and emotional ones. Neuropathy may result in amputation through diferent ways including loss of one or more funtions in peripheral nerves.

Glycaemic control, symptom control and feet care are standard measures that affect all health professionals implied in diabetic patients attention. Deeper knowledge of pathogenic mecanisms should result in new treatments which seem to be more effective if administered early, when abnormalities are more likely to be reversible.

KEY WORDS: Diabetes mellitus. Diabetic foot. Painful neuropathy. Aetiology. Treatment.

Martín Muñoz MC, Albarrán Juan ME, Lumbreras Marín EM. Revisión sobre el dolor neuropático en el síndrome del pie diabético. An Med Interna (Madrid) 2004; 21: 450-455.

\section{INTRODUCCIÓN}

La diabetes mellitus (DM) es una enfermedad metabólica crónica y compleja que se caracteriza por deficiencia absoluta o relativa de insulina, hiperglucemia crónica y otras alteraciones del metabolismo de los carbohidratos y de los lípidos; ello a su vez puede originar múltiples complicaciones microvasculares en los ojos, el riñón y las extremidades inferiores, así como neuropatías periféricas $\mathrm{y}$, frecuentemente, lesiones macrovasculares y coronarias (1).

El pie diabético, en particular, se define como la infección, la ulceración y la destrucción de los tejidos profundos, asocia- do a alteraciones neurológicas (pérdida de la sensibilidad al dolor) y vasculopatía periférica de diversa gravedad en las extremidades inferiores (1).

Las afecciones de los pies en los pacientes con DM constituye una de las principales causas de morbilidad y discapacidad, con importante repercusión biológica, psicológica y social, pues disminuye su calidad de vida. Un rápido reconocimiento y una adecuada atención de los factores de riesgo que condicionan el pie diabético pueden prevenir o retardar la aparición de complicaciones. Esto no sólo es una tarea del endocrinólogo, el neurólogo y el cirujano vascular, sino que involucra a todo el equipo de salud dedicado a la atención

Trabajo aceptado: 5 de abril de 2004 
integral de los pacientes con DM; o sea, a los médicos comunitarios, las enfermeras, los especialistas en nutrición, los educadores, los podólogos y los psicólogos. Únicamente con este enfoque podremos minimizar las consecuencias de la DM y sus complicaciones (2).

El proceso de producción y generación del dolor neuropático es diferente de los mecanismos homeostáticos fisiológicos normales (3), se presenta como un dolor crónico o un dolor persistente, que no posee sólo causas biológicas sino que están involucradas respuestas físicas y emocionales (4).

La DM es la causa más común de neuropatía en el mundo occidental. Se ha estimado una prevalencia del 28,5\% en estudios realizados en Estados Unidos (5). Esta prevalencia varía en función de las medidas utilizadas para definir la neuropatía diabética. Usando una definición basada en los síntomas, signos, estudios de conducción nerviosa, test de sensibilidad cuantitativa y test autonómicos, la neuropatía estaba presente en un $66 \%$ de los pacientes diabéticos. Dicha prevalencia se incrementa con la duración de la diabetes y está en relación con el mal control metabólico en pacientes ancianos. La neuropatía diabética es un grupo muy heterogéneo que puede incluir tanto las mono o polineuropatías como las plexopatías o las radiculopatías (5).

Las definiciones utilizadas por la ADA (Asociación Americana de Diabetes) para el diagnóstico de la DM (6) quedan resumidas en la tabla $\mathrm{I}$.

\section{TABLA I}

DEFINICIONES USADAS PARA EL METABOLISMO ANORMAL DE LA GLUCOSA

\begin{tabular}{ll}
\hline Diabetes & Síntomas de diabetes asociados con una cifra \\
& casual de glucosa $>0=200 \mathrm{mg} / \mathrm{dl}(11,1$ \\
& $\mathrm{mmol} / \mathrm{l})$ o una glucemia basal $>0=126 \mathrm{mg} / \mathrm{dl}$ \\
& $(7,0 \mathrm{mmol} / \mathrm{l}) 02 \mathrm{~h}$ después de comer glucemia $>$ \\
& $0=200 \mathrm{mg} / \mathrm{dl}(11,1 \mathrm{mmol} / \mathrm{l})$ durante un test de \\
& tolerancia oral a la glucosa.
\end{tabular}

Tolerancia anormal a la glucosa Cifras de glucosa 2 h después de la ingesta $>0=$ $140 \mathrm{mmol} / \mathrm{dl}(7,8 \mathrm{mmol} / \mathrm{l})$ y $<200 \mathrm{mg} / \mathrm{dl}(11,1$ $\mathrm{mmol} / \mathrm{l})$

Riesgo elevado de diabetes Determinación de glucemia rápida $>0=110$ $\mathrm{mg} / \mathrm{dl}(6,1 \mathrm{mmol} / \mathrm{l}) \mathrm{y}<126 / \mathrm{dl}(7 \mathrm{mmol} / \mathrm{l})$

\section{ETIOLOGÍA Y PATOGENIA}

Al pie diabético se agregan problemas potenciales de los huesos y articulaciones debido a la insuficiencia vascular periférica y la neuropatía. El control de la glucemia parece prevenir el desarrollo de microangiopatías de la retina y el riñón y probablemente retarda la evolución de neuropatías, pero no parece tener suficiente efecto sobre la macroangiopatía. La insuficiencia vascular periférica conduce a la necrosis y la amputación. Muchas lesiones del pie diabético se inician con síntomas de neuropatía y, si aumentan las parestesias y disminuye la sensación de dolor, los traumatismos subsecuentes y repetidos producen lesiones mayores que, asociadas a la insuficiencia vascular, impiden la cicatrización. La neuropatía puede ocasionar degeneración articular (neuropatía de Charcot) y dar por resultado nuevos apoyos del pie que reciclan el problema de úlcera-infección (7).
Hay muchas clasificaciones clínicas de la neuropatía diabética. Una revisión sobre las teorías patogénicas del dolor sensomotor neuropático refleja que los estudios referentes al estado de desmielinización temprana, han sido sustituidos por un robusto modelo predictivo, en que la degradación inicial tiene mínimos efectos en la velocidad de conducción nerviosa (8). Hay investigaciones que demuestran que la afectación de tipos de fibras nerviosas tanto aferentes (sensoriales) como eferentes (motoras) pueden no estar uniformemente afectadas (9). Las fibras nerviosas periféricas simpático-adrenérgicas y colinérgicas tienen alteraciones simultaneas tempranas en pacientes diabéticos, aun cuando no sea evidente la neuropatía clínicamente (10). Las definiciones de los tipos más comunes de dolor se muestran en la tabla II.

\section{TABLA II}

\section{DEFINICIONES DE TIPOS COMUNES DE DOLOR}

\begin{tabular}{ll}
\hline Terminología del dolor & Definiciones comúnmente aceptadas \\
\hline Dolor reflejo & $\begin{array}{c}\text { Dolor en un área lejana del sitio donde se encuentra } \\
\text { la agresión tisular }\end{array}$ \\
Dolor fantasma & $\begin{array}{c}\text { Dolor en una parte del cuerpo que ha sido quirúrgica- } \\
\text { mente extirpada o se encuentra ausente (congénito) } \\
\text { Alodinia }\end{array}$ \\
Hiperalgesia & $\begin{array}{c}\text { Dolor exacermulo normal es percibido como doloroso } \\
\text { Sensibilización }\end{array}$ \\
& $\begin{array}{c}\text { Los receptores responden con gran intensidad a un } \\
\text { estímulo o responden a un estímulo cuando no lo } \\
\text { harían en condiciones normales }\end{array}$ \\
Hiperpatía & Dolor intenso con estímulos repetidos \\
Dolor evocado por estímulos & Dolor producido tras un estímulo después del daño o \\
& alteración en las neuronas sensoriales
\end{tabular}

El daño isquémico microvascular tiene relación con el daño producido a las estructuras de fibras nerviosas (5). La combinación de la neuropatía sensorial y la isquémica tiene efecto directo adverso sobre los mecanismos de defensa del huésped; en particular hace a los pacientes con DM mas vulnerables a las infecciones del pie. La neuropatía puede conducir a la amputación por varios caminos, los cuales incluyen la perdida de la función autonómica, sensitiva y motora de los nervios periféricos (11). En la figura 1 se presenta un esquema simplificado de la fisiopatología de la neuropatía en el pie diabético y sus consecuencias.

La neuropatía en diabéticos puede ser también causada, en parte, por déficit en la regeneración de las fibras nerviosas, mecanismo apoyado por anormalidades en las repuestas tempranas de los genes necesarios para el inicio de la regeneración de las fibras (12). El calcio y los canales del calcio también parecen tener un papel .

\section{FACTORES DE RIESGO DEL PIE DIABÉTICO}

Los factores de riesgo para el desarrollo del síndrome del pie diabético incluyen aquellos relacionados con la aparición de úlceras y la amputación del pie, ya que las primeras constituyen una expresión del síndrome y la amputación es su com- 


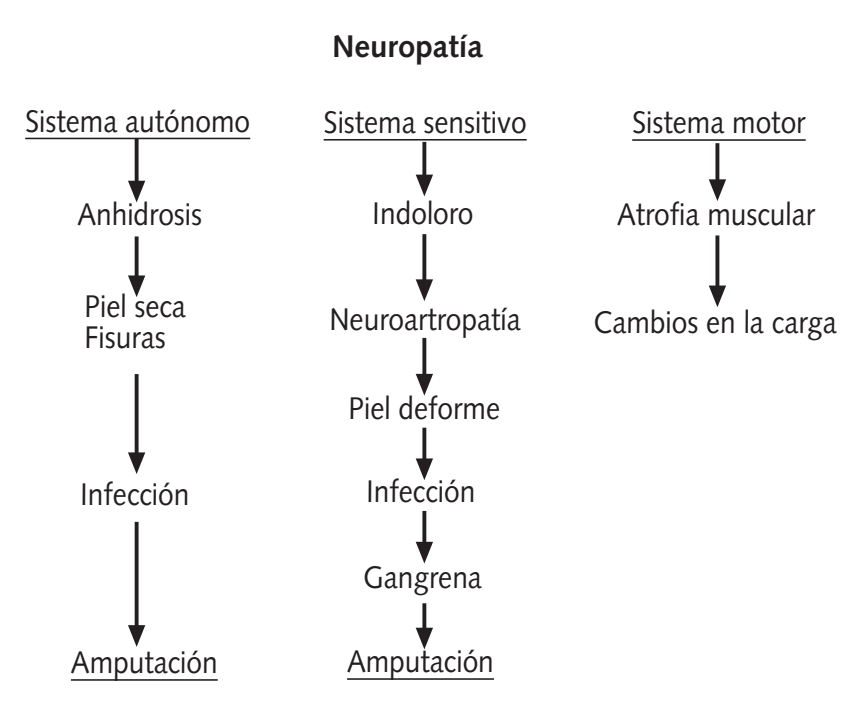

Fig. 1. Fisiopatología del síndrome del pie diabético.

plicación mas frecuente. Según la posibilidad de intervenir sobre ellos y eliminarlos, pudiéramos dividirlos en factores de riesgo modificables y no modificables (13).

\section{Factores modificables}

—Descontrol metabólico

-Factores sociales

-Deformidades anatómicas

- Aumento de la presión plantar

- Calzado inadecuado

- Sobrepeso corporal u obesidad

-Alcoholismo

- Tabaquismo

—-Ingestión de betabloqueantes

\section{No modificables}

- Edad

- Tiempo de evolución de la diabetes

-Sexo

-Antecedentes de ulceras y/o amputaciones

-Antecedentes de neuropatía, angiopatía, retinopatía y nefropatía (pudieran ser modificables en estadios iniciales)

-Limitación de los movimientos articulares

\section{CLÍNICA DEL PIE NEUROPÁTICO}

Los signos y síntomas de la neuropatía del pie diabético son: temperatura conservada, puede haber parestesia, hiperestesia, hipoestesia, dolor radicular, perdida de reflejos osteotendinosos, perdida de la sensación vibratoria y de posición, atrofia muscular, dilataciones venosas, anhidrosis, formación de callos en puntos de presión, ulceras tróficas generalmente en el talón, maléolo, cara superior de articulación de los dedos, cabeza del quinto y primer metatarsianos, ápice de lo dedos; tiene borde circular con callo alrededor. También pue- de haber infección, cambios de talla-deformación del pie-plano-valgo-varo, desmineralización, osteolisis y articulación de Charcot. La neuropatía es frecuentemente bilateral. Las parestesias, a veces paroxísticas, se refieren como dolor o como una sensación confusa de ardor o quemadura (14).

La lesión inicial puede ser un mal perforante plantar, asociado o no a sepsis. Puede haber osteoartropatía, que son los cambios destructivos óseos asépticos presentes en el pie de un paciente con DM. También las articulaciones pueden afectarse y se asocia a deformación podálica. Desde el punto de vista clínico puede comenzar de forma repentina con signos inflamatorios en las porciones distales de un pie o tobillo, a menudo con una historia de trauma menor, o como una artropatía lenta y progresiva con inflamación insidiosa en el transcurso de meses o años. En el pie hay caída del arco medio y prominencias óseas en zonas peculiares. Es relativamente dolorosa y puede ser bilateral. En ocasiones se acompaña de fracturas patológicas (14).

\section{DIAGNÓSTICO}

La neuropatía diabética es la complicación mas frecuente y precoz de la diabetes. A pesar de ello suele ser la más tardíamente diagnosticada. Su prevalencia es difícil de establecer debido a la ausencia de criterios diagnósticos unificados, a la multiplicidad de métodos diagnósticos y a la heterogeneidad de las formas clínicas. Su evolución y gravedad se correlacionan con la duración de la enfermedad y el mal control metabólico.

Las herramientas mas utilizadas hoy en el cribado de la neuropatía, la principal complicación que origina el pie diabético, es el Monofilamento de Semmens-Weinstein y el diapasón de $128 \mathrm{Mhz}$. Otra de las escalas utilizadas es el Michigan Neuropathy Screening Instrument, un diagnóstico de ocho puntos que incluye el examen clínico de los pies, la presencia o ausencia de ulceraciones en los mismos, el diagnóstico de la sensación vibratoria en el primer dedo y la gradación de los reflejos de la rodilla (5) (Tabla III).

Otros métodos de diagnóstico son la cuantificación de los síntomas usando instrumentos como "puntuación de síntomas neuropáticos" y "perfiles de síntomas neuropáticos". Han sido puntuados como síntomas positivos: sensaciones eléctricas, pinchazos, dolor, prurito, quemazón, sensibilidad vibratoria, etc., considerados como clínica primaria que refieren los pacientes y pueden ser referencia para estudios epidemiológicos y control del proceso clínico (15).

La calidad de vida puede también servir para el diagnóstico, particularmente para establecer comparaciones entre ésta y los métodos de medida convenciones de neuropatía (16).

\section{DIAGNÓSTICO DIFERENCIAL DEL PIE DIABÉTICO}

Debe hacerse cuando hay presencia de alcoholismo, hernia de disco, intoxicación por metales y drogas, colagenosis, anemia perniciosa, neoplasias, uremia, y lepra, entre otras. La alteración neuropática mas importante en el pie diabético es la perdida de la sensibilidad, por lo que el pie queda expuesto a traumatismos indoloros mecánicos, químicos o térmicos. Es muy frecuente que los problemas del pie diabético se inicien a causa de la "cirugía domestica", es decir, cuando el paciente 
TABLA III

EXAMEN CLÍNICO BREVE PARA DESPISTAJE PROPUESTO POR EL GRUPO DE MICHIGAN

(Cada pie aporta un puntaje por separado)

\begin{tabular}{llll}
\hline & & Puntaje & \\
\hline Apariencia del pie & 0 & 0,5 & 1 \\
& Normal & & $\begin{array}{l}\text { Deformado } \\
\text { Piel seca } \\
\text { Infección, fisuras }\end{array}$ \\
Ulceración & Ausente & & Presente \\
Reflejo aquileo & Presente & Presente con refuerzo & Ausente \\
Percepción de vibración & Presente & Disminuida & Ausente \\
La probabilidad de neuropatía es alta si el puntaje es $>2 / 8$ & (sensibilidad 80\%, especificidad 95\%) \\
\hline
\end{tabular}

se recorta las uñas y los callos hasta planos muy profundos porque ya no tiene sensibilidad en esa región, lo que predispone al paciente a adquirir la infección mas fácilmente. El diabético puede empezar a provocarse lesiones o a sentir manifestaciones de ellas, al intentar calentar los pies con agua caliente o cojines eléctricos, como respuesta a la sensación de pie frío. Por otro lado el uso incorrecto de un zapato que obliga al apoyo plantar defectuoso, produce lesiones traumáticas, sobre todo si la neuropatía ya produjo neuroartropatías; la consecuente desviación del eje de los huesos deforma el pie, aumenta su diámetro y lo ensancha. Con la utilización del mismo calzado agresor, el cual es tolerado por la insensibilidad, se inicia un círculo vicioso. La situación se agrava con edema, inflamación e infección a veces dolorosa. La posición deformada de los dedos es común: dedos en garra o gatillo, sobre todo si los músculos intrínsecos del pie se han afectado; a su vez estas deformaciones producen nuevas callosidades (17).

\section{PREVENCIÓN Y TRATAMIENTO}

\section{CONTROL METABÓLICO}

Se han asociado cambios rápidos en el control metabólico como desencadenantes del dolor neuropático diabético. Los niveles elevados de glucosa y las fluctuaciones en las cifras están relacionados con síntomas dolorosos18. Parece ser que controles intensivos de la glucemia reducen la prevalencia y progresión de la neuropatía periférica. Estudios morfológicos sugieren que la severidad de la polineuropatía diabética está estrechamente relacionada con el control glucémico tanto en individuos que padezcan diabetes tipo 1 como diabetes tipo 2 $(19,20)$.

Estudios realizados en Estados Unidos demuestran que una gran proporción de pacientes ancianos con DM no reciben un seguimiento y tratamiento en concordancia con las recomendaciones de la $\mathrm{ADA}$; no obstante, en los últimos años, el grado de intervención en el cuidado se ha incrementado para llegar a un mejor control metabólico de estos pacientes (20).

Un estudio multidisciplinar comparativo entre 2 grupos de diabéticos (grupo control y un grupo con seguimiento por Atención Primaria-especializada) ha mostrado que, tras 6 meses, los niveles de control de glucemia (HbA1c) son significativamente menores en aquellos que han tenido un seguimiento y que han sufrido menor número de ingresos hospitalarios. La combinación de materias educativas, revisión de los roles profesionales y educación del paciente resultan significativamente importantes en el buen control de la glucemia (20). Es fundamental la realización de un tratamiento precoz, para la estabilización, preferiblemente reversible de la neuropatía diabética (5).

\section{TERAPIAS FARMACOLÓGICAS}

No existen actualmente tratamientos que restauren la función nerviosa, están orientados principalmente a paliar los síntomas, especialmente el dolor severo. Normalmente las lesiones de larga evolución requieren la prescripción de agentes farmacológicos sistémicos o tópicos.

Entre los agentes farmacológicos utilizados destacan:

1. Antidepresivos

-Antidepresivos tricíclicos.

Desde 1970 los antidepresivos tricíclicos han representado el tratamiento de primera línea para el dolor neuropático periférico (21). Los estudios han mostrado que estos agentes promueven la analgesia a nivel térmico, mecánico y eléctrico en pacientes diabéticos (22), siendo su acción anticolinérgica el principal efecto secundario (23). Algunos autores sugieren que los antidepresivos tricíclicos deben ser prescritos para síntomas de dolor urente, aunque no existe una asociación directa entre esta manifestación particular del dolor y la eficacia del fármaco (9).

—Inhibidores de la recaptación de serotonina.

Su modo de acción está basado en observaciones experimentales a cerca del papel en la analgesia que presentan como mediadores. Hay evidencias sobre la reducción del dolor lacerante al utilizar la paroxetina (9).

2. Anticonvulsivantes

-Gabapentina.

Farmacológicamente parece no tener un efecto directo sobre el metabolismo del GABA. Parece ser que es un inhibi- 


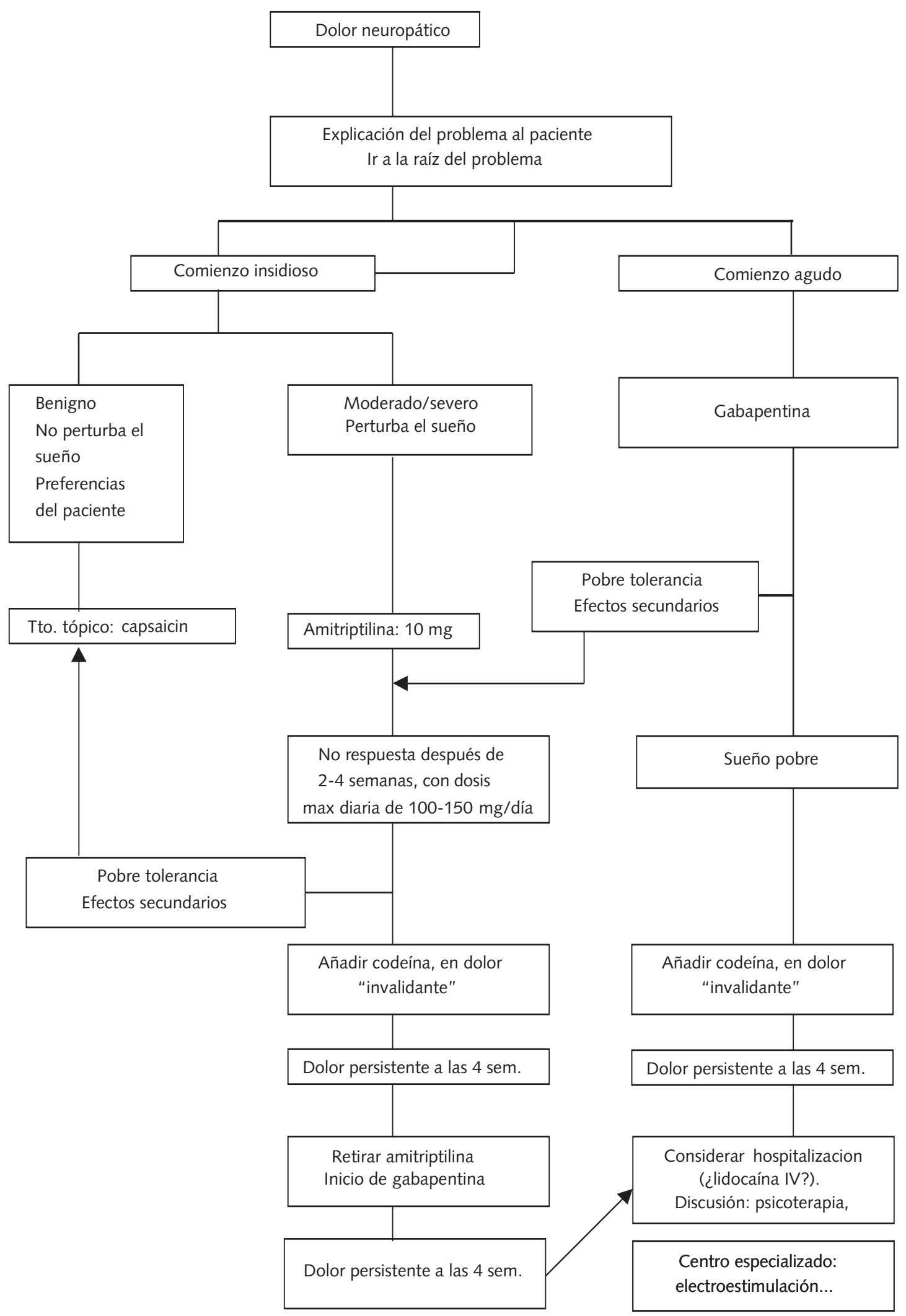

Fig. 2. Selección del tratamiento para el dolor neuropático en diabetes mellitus. 
dor de la activación del voltaje del calcio y de los canales del sodio sugiriendo un efecto analgésico sobre niveles de la médula espinal (24). La gabapentina suele ser bien tolerada a dosis de $1.800 \mathrm{mg} /$ día que se han mostrado efectivas.

Otros fármacos: carbamacepina, fenitoina, lamotrigina, topiramato, etc.

\section{Antiarrítmicos}

4. Tratamientos tópicos

- Capsaicin. Su efecto terapéutico es acumulativo lográndose el máximo efecto en 4-6 semanas (25).

5. Antagonistas NMDA: dextrometorfano

6. Analgésicos narcóticos

El rango de opiáceos prescrito incluyen la codeína, tramadol y metadona. Los pacientes deben revisarse regularmente para el control del dolor y recordar el riesgo de dependencia y los efectos secundarios de este tipo de fármacos.

7. Anttiinflamatorios no esteroideos

8. Terapias no farmacológicas

- Terapias de estimulación nerviosa

Incluyen TENS (estimulación transcutánea eléctrica nerviosa) y PENS (estimulación percutánea eléctrica nerviosa) y acupuntura (9).

-Estimulación eléctrica de la médula espinal

Al ser un procedimiento invasivo, se reserva a pacientes con dolor refractario al tratamiento convencional.

- Consejo y tratamiento psicológico

Consiste fundamentalmente en técnicas para el control del dolor y de relajación.

\section{CONCLUSIONES}

La neuropatía diabética es una patología de gran morbilidad. El control de la glucemia, de los síntomas, el cuidado de los pies son estándares de referencia para la intervención sanitaria. Como quiera que sea, el incremento en el conocimiento de los mecanismos patogénicos abre una puerta para futuros tratamientos. Probablemente sean éstos más efectivos cuanto más tempranamente se apliquen en el curso de la neuropatía, cuando la afectación nerviosa es reversible todavía.

El seguimiento e intervenciones múltiples por parte de Atención Primaria son fundamentales en la mejoría de los síntomas y del estado de salud general en estos pacientes, viéndose incrementada la adherencia a los tratamientos. La intervención, el desarrollo y la evaluación de programas de salud para la prevención, el control y la vigilancia de la enfermedad cardiovascular, la enfermedad vascular periférica, la DM y el pie diabético, demandan programas de adiestramiento para médicos, enfermeras, técnicos, gerentes y estudiantes.

El deterioro de la agudeza visual y la ausencia de sensaciones, con frecuencia son resultado del exceso de confianza que depositan los pacientes en los profesionales de la salud para que las detecten.

Las opciones para el tratamiento del pie neuropático e isquémico varían en complejidad. La evaluación adecuada y la identificación oportuna de los signos clínicos de úlceras neuropáticas e isquémicas asegurará un buen pronóstico y la mejor intervención para el tratamiento del pie diabético.

\section{Bibliografía}

1. Eastman RC, Vinicor F. Science: moving us in the right direction. Diabetes Care 1997; 20: 1057-1058.

2. Sumpio BE. Foot ulcers. N Engl J Med 2000; 14: 787-793.

3. Serra J. Overview of neuropathic pain síndromes. Acta Neurologia Scand 1999; 173: 7-11.

4. Woolf CJ, Mannion RJ. Neuropathic pain: aetiology, symptoms, mechanisms and management. Lancet 1999; 353: 1959-1964.

5. Simmons Z, Feldman EL. Updata on diabetic neuropathy. Current Opinion in Neurology 2000; 15: 595-603.

6. American Diabetes Association. Standards of medical care for patients with diabetes mellitus. Diabetes Care 2002; 25(Suppl. 1): S33-S49.

7. Stess RM, Jensen SR, Mirmiran R. The role of dynamic plantar pressures in diabetic foot ulcers. Diabetes Care 1997; 20(5): 855-858.

8. Wang Y, Bowersox SS, Pettus M, Gao OA. Antinociceptive properties of fenfluramine, a serotonin reuptake inhibitor, in a rat model of neuropathy. J Pharmacol Exp Therapeutics 1999; 291: 1008-1016.

9. Spruce MC, Potter J, Coppini DV. The pathogenesis and management of painful diabetic neuropathy: a review. Diabet Med 2003; 20: 88-98.

10. Cacciatori V, Dellera A, Bellavere F, Bongiovanni LG, Teatini F, Gemma ML. Comparative assessment of peripheral sympathetic function by postural vasoconstriction arteriolar reflex and sympathetic skin response in NIDDM patients. Am J Med 1997; 102 (4): 365-370.

11. Lopez-Antuñano S, Lopez-Antuñano FJ. Diabetes Mellitus y lesiones del pie. Salud Publica de México 1998; 40 (3): 281-292.

12. Xu G, Sima AAF. Altered immediate early gene expresión in injured diabetic nerve: implications in regeneration. J Neuropathol Exp Neurol 2001; 60: 972-983.

13. Sibbald RG, Browne AC. Diabetic foot ulcers. Can J Diabetes Care 2000; 24: 41-49.

14. Sell Lluveras JL, Miguel Domínguez I. Guía práctica para el diagnóstico

y el tratamiento del síndrome del pie diabético. Rev Cubana Endocrinol 2001; 12(3): 188-197.

15. Apfel SC, Asbury AK, Bril V. Positive neuropathic sensory symptoms as endpoints in diabetic neuropathy trials. J Neurol Sci 2001; 189: 3-5.

16. Padua L, Aprile I, Saponara C. Multiperspective assessment of peripheral nerve involvement in diabetic patients. Eur Neurol 2001; 45: 214 221.

17. Thomas PK. Classification, differential diagnosis, and staging of diabetic peripheralneuropathy. Diabetes 1997; 46 suppl 2: S54-S57.

18. Wunderlich RP, Peters EJG, Bosma J, Armstrong DG. Pathophysiology and treatment of painful diabetic neuropathy of the lower extremity. South Med J 1998; 91: 957-960.

19. Perkins BA, Greene DA, Bril V. Glycaemic control is related to the morphological severity of diabetic sensorimotor polyneuropathy. Diabetes Care 2001; 24: 748-752

20. Renders CM, Valk GD, Griffin S, Wagner EH, Eijk JThM van, Assendelft WJJ. Interventions to improve the management of Dabetes Mellitus in Primary Care, outpatient and community settings. The Cochrane Library, Issue 3, 2003.

21. Collins SL, Moore A, McQuay HJ, Wiffen P. Anti-depressants and anticonvulsants for diabetic neuropathy and postherpetic neuralgia: a quantitative systemic review. J Pain Symptom Manage 2000; 20: 449-458.

22. Nash TP. Treatment options in painful diabetic neuropathy. Acta Neurologica Scand 1999; 173: 36-42.

23. Backonja MM, Beydoun A, Edwards KR, Schwartz SL, Fonseca V, Hes M. Gabapentin for symptomatic treatment of painful neuropathy in patients with diabetes mellitus. J Am Med Assoc 1998; 280: 1831-1836.

24. Backonja MM. Gabapentin monotherapy for the symptomatic treatment of painful neuropathy: a muti-centre, double-blind, placebo-controlled trial in patients with diabetes mellitus. Epilepsia 1999; 40: 557-559. 